\title{
Eliminating the mystery from the concept of emergence
}

\author{
Brian R. Johnson
}

Received: 30 August 2009/ Accepted: 9 August 2010/Published online: 11 September 2010

(C) The Author(s) 2010. This article is published with open access at Springerlink.com

\begin{abstract}
While some branches of complexity theory are advancing rapidly, the same cannot be said for our understanding of emergence. Despite a complete knowledge of the rules underlying the interactions between the parts of many systems, we are often baffled by their sudden transitions from simple to complex. Here I propose a solution to this conceptual problem. Given that emergence is often the result of many interactions occurring simultaneously in time and space, an ability to intuitively grasp it would require the ability to consciously think in parallel. A simple exercise is used to demonstrate that we do not possess this ability. Our surprise at the behaviour of cellular automata models, and the natural cases of pattern formation they mimic, is then explained from this perspective. This work suggests that the cognitive limitations of the mind can be as significant a barrier to scientific progress as the limitations of our senses.
\end{abstract}

Keywords Emergence - Complexity theory · Pattern formation ·

Cellular automata $\cdot$ Parallel processing

\section{Introduction}

The study of complex systems is one of the few areas where physical and life scientists share a common focus of study. If for no other reason, this makes it an intriguing subject. When the same principles can be used to model ecological communities, patterns of animal coat coloration, and the complex dynamics of chemical and physical systems one can only conclude that deep principles are being explored (Prigogine 1980; Nicolis and Prigogine 1989; Bonabeau et al. 1997; Camazine et al.

B. R. Johnson $(\bowtie)$

Department of Environmental Science, Policy \& Management, University of California,

Berkeley, 245 Hilgard Hall, MC3114, Berkeley, CA 94720-3114, USA

e-mail: bjohnson741@berkeley.edu 
2001; Vicsek 2002; Coveney 2003). While great progress has been made in understanding some branches of complexity theory, such as self-organization, the same cannot be said of our understanding of the concept of emergence (Crutchfield 1994; Corning 2002).

There seem to be three basic approaches to defining and exploring emergent properties. One approach seeks to define the concept using informal logic. These researchers focus on the general scope of reductionism (reviewed in Newth and Finnigan 2006 and Halley and Winkler 2006). A consensus view might be that complex systems are irreducible and emergence occurs when a whole is more than the sum of its parts and their interactions. A second approach essentially defines emergence by showing that it is the result of a particular process such as symmetry breaking (Anderson 1972) or self-organization (Turing 1952; Prigogine 1980; Nicolis and Prigogine 1989; Camazine et al. 2001). This approach has had the greatest success, in that we now understand some of the dynamics of systems that show emergence. The third approach seeks to provide an explicit set of mathematical equations for quantifying the degree of complexity in any system such that a comparison of the amount of information in the parts can be compared to that in the whole (Crutchfield and Young 1989; Nehaniv and Rhodes 2000; Adami 2002; Bar-Yam 2004). These studies attempt to predict when complex phenomena will result from interacting units with varying degrees of information.

While all of these approaches are valuable for addressing different aspects of emergence, they fail to give us a satisfying explanation for the aspect of it that inspires the most curiosity and confusion. I am referring to those situations where we possess a complete knowledge of the simple rules governing the behaviour of a number of interactants and, nevertheless, our intuition is still baffled by the sudden onset of pattern from disorder. This form of emergence generates much of the interest in the topic, as it inspires fascination and a promise of major advances for some, while its mysterious nature is a source of frustration for others.

In this paper, I will argue for a new conceptual way to view emergent properties that eliminates their mysterious nature. The primary goal is to address the surprise that emergent phenomena evoke. I will argue that emergence is surprising and unpredictable because many interactions occurring simultaneously in space and time are the basis for rapid transitions between order and disorder. Further, the fact that we cannot consciously think in parallel explains our bewilderment when pondering the outcome of such processes.

\section{Parallel conscious thinking?}

The following argument requires that the reader participate in a simple exercise. Carefully attempt the tasks in Fig. 1. There is nothing complex here. You are asked to add together lists of ones and zeros. Moreover, since the zeros do not count, adding is not even necessary. All that is required is to count the ones as you scan across the line. People vary in the rate at which they can accomplish this, as they do for most mental exercises. What happens, however, when one attempts to count more than one row simultaneously? Although we can do two, it is only by adding 


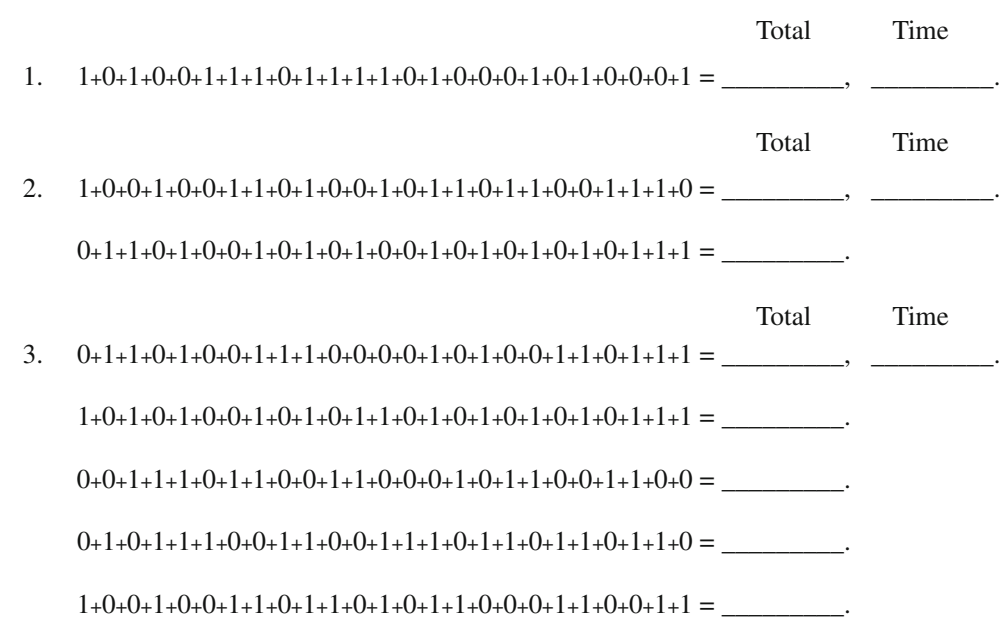

Fig. 1 A simple exercise designed to test your ability to consciously think in parallel. Add the numbers in part 1 carefully and record the time in takes. Now try to do the same for part 2 by scanning across both lines at the same time and adding in parallel. An ability to add in parallel would allow you to complete part 2 in the same time it took you to complete part 1 . Now try part 3 . The reader is encouraged to invent other such games and test themselves in other contexts. Our inability to add more than one line at a time has nothing to do with our ability to see both lines. It is clearly cognitive

the first two numbers in the first row, then remembering that result and moving to the second line and doing the same and then iterating back and forth that we can do so. Counting two rows in parallel is equivalent to reading two sentences at once. This is functionally impossible for us. Personally, I cannot even begin the task of simultaneously counting five rows. I do not know the physiological reason, of course, but my mind (and the mind of everyone I have asked to attempt this task) seems to have no capacity for it whatsoever. It may, thus, be a hardware problem and not simply a problem with how we are taught to add and subtract numbers.

Consider the relationship between the tasks of adding one row of numbers and adding two rows simultaneously. I chose lines of ones and zeros to make the point that the difficulty of the first task has nothing to do with our inability to do the second. We perform many tasks in sequence that are much more difficult than the adding of two rows of numbers in parallel. Further, our visual system is capable of just this sort of parallel processing (otherwise we could not see over a sufficient area in time so as be surprised by the rapidity of pattern formation in nature). The brain is in fact capable of parallel processing in many contexts (Balleine and Killcross 2006; Dieck and Brandstatter 2006; Spehr et al. 2006). We simply may not have evolved the ability to consciously think in parallel because it is unnecessary in our daily lives.

\section{Multiple simultaneous interactions}

Multiple simultaneous interactions are those in which each interactant is responsive to more than one other at each moment in time. Imagine an orderly front of 
interacting units in which each receives feedback from all its neighbours as they move forward in tandem. The suggestion that this type of interaction leads to emergence is fundamentally different from noting that we are often overwhelmed with enormous amounts of information. Gas particles, for example, exist in vast quantities, and interact millions of times within a very short time span. Of course, with respect to the movement of one molecule, we cannot follow such a long causal trajectory. This is independent of the phenomenon of which I am speaking, however. When interactions are multiple and simultaneous, they need not be many to overwhelm our cognitive ability. Figure 1 makes this point in one context and I could propose many others. I will not labour the point here, however, since I will explain a well known case of this phenomenon in the next section. It is important here to grasp that in nature, and many of our best models for exploring nature, operations are not binary and mentally following even a handful of simultaneous interactions may be beyond our intuitive capabilities.

\section{Cellular automata}

The cases of emergence in nature are many and varied (Camazine et al. 2001; Coveney 2003). Instead of exhaustively discussing individual cases, I will focus on one abstraction, the cellular automata model, which can be used to model any case of pattern formation (Wolfram 1984a, b). Further, the complex behaviour of these models captures the surprising and mysterious nature of emergence. In short, if we can understand why we cannot intuitively predict the behaviour of cellular automata, we will have made progress toward understanding why we lack the ability to do so for many natural phenomena.

Figure 2 shows several generations of Conway's game of life (Gardner 1970, 1971; reviewed in Aleksic 2000). The environment of the model is an area divided into many grid squares. Each square is surrounded by eight other squares. The squares can be in two states: living or dead (grey or black in the figure). The rules for determining whether a site lives or dies between generations are the following: (1) living sites with two living neighbours remain alive, (2) any site with three living neighbours either stays alive or is born, and (3) sites either die or remain dead in all other circumstances. This model has fascinating behaviour. Given a random starting condition, sometimes all the grids die quickly, sometimes they live but show random patterns of movement, and sometimes they form geometric shapes that float across the grid. For most initial states, a person is incapable of predicting which of these possibilities will occur. The spatial and temporal patterns the game of life produces seem beyond the simplicity of the rules that govern the process. The game thus displays the surprising character of emergence. Let us now explore this model from the perspective of our inability to consciously think in parallel.

The key to the problem is to recognize that the computer must acquire a global property of a focal square's neighbours before making a decision. The basic operations, therefore, though simple, require that each cell receive input simultaneously from many others. This is necessary, of course, because any of the 8 neighbours of a square can vary from one time to step to the next. Thus, the computer 


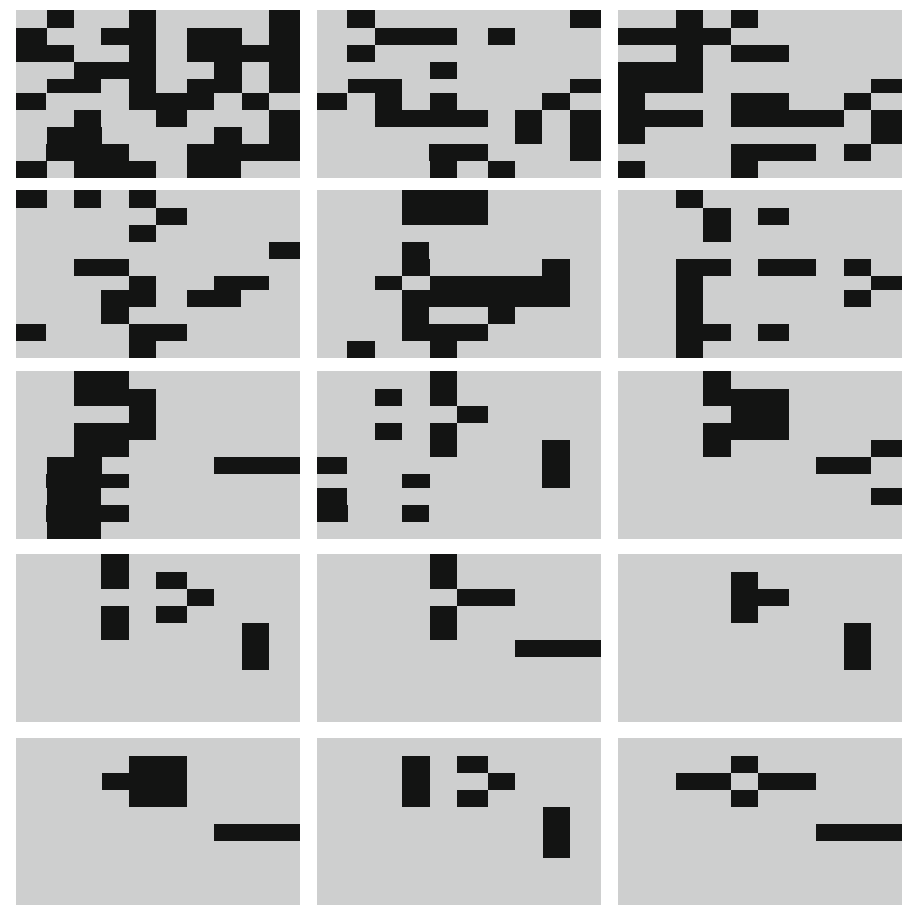

Fig. 2 Several generations (left to right) of Conway's game of life. The grids live or die (turn black or grey) depending on the following rules (1) living sites with two living neighbours remain alive, (2) any site with three living neighbours either stays alive or is born, and (3) sites either die or remain dead in all other circumstances. The game is capable of producing complex patterns that seem, to our intuition, far beyond its simple rules. I argue that we are unable to predict the outcome of such games due to our inability to consider the behaviour of multiple grids in parallel. If we could accomplish the adding tasks in Fig. 1, we would not consider the game of life's behaviour surprising

is programmed to roughly approximate multiple simultaneous interactions by storing the information from several binary operations into one global parameter before using that global parameter alone to perform an operation. Of course, in nature there can be any number of interactants interacting simultaneously and the rules governing their behaviour can be complex, but, hopefully, it is intuitively clear that the cellular automata model coarsely simulates a process of multiple simultaneous interactions.

Let us now consider why we perceive cellular automata behaviour as surprising and unpredictable. First, anyone knowing the rules can look at one cell and easily predict what it will do at the next time step. The problem for predicting time step 2 is that you would also need to know the state of each of the 8 neighbours at time step 2 and they all will have changed in accordance with the properties of their neighbours and so on and so on throughout the grid. Hence, the problem requires the same ability to think in parallel that Fig. 1 demonstrates we do not possess. Of course, it does not really matter how many neighbours there are any more than it matters how many lines of 1 and 0s one is asked to add. We cannot think in parallel at all, so three, eight, or a million simultaneous interactions will all appear equally baffling. 


\section{Conclusion}

Despite a complete knowledge of the rules underlying the interactions between the parts of many systems we are nevertheless baffled by their sudden transitions from simple to complex, random to ordered, and ordered to random (Wolfram 2002). This paper argues that this limitation is a product of the way our minds organize the complexity of natural interactions into sequences of binary operations. Much of what we term emergence is, consequently, a subjective product of our intuitive methods of problem solving and not a characteristic of nature that requires explanation beyond that explicit in the algorithms we use to generate the behaviour with computer simulations.

Kant pointed out that because we perceive nature through a filter (the mind) it precludes any objective understanding of its properties (Russell 1945). Scientists have largely ignored this problem; and like most scientists, I believe we have done so with good reason. For example, we cannot directly perceive microbes and when Hooke and van Leeuwenhoek built the first microscopes and notified us of their existence, the discovery was met with great surprise and resistance. By the time von Frisch (reviewed in Frisch 1967) informed us that honey bees navigate using patterns of polarized light, no one was shocked that an invisible wealth of information existed right before our eyes. We had come to think of our sensory equipment as a product of our evolutionary history and not an objective means of perceiving the world. The analogous fact, however, that our cognitive mechanisms reflect an evolutionary history that may preclude a direct intellectual experience of natural phenomena is not yet widely appreciated (Crutchfield 1994). In a nutshell, we may find that we need to augment our natural intellectual abilities with help from parallel processing computers, in much the same way as we augment our sensory abilities with various gadgets, if we are to both describe and understand the complexity of nature (Vicsek 2002).

Hopefully, this paper will have impressed upon the reader that understanding the way our cognitive limitations affect the construction of the logical languages we use to understand nature should be a major goal of science. As this is already a goal of many researchers working on similar questions in cognitive science (Pinker 1997; Ramachandran and Blakeslee 1998; Dehaene et al. 1999; Berent et al. 2005) all that is required is that every branch of science becomes cognizant of these issues.

Acknowledgments Nigel Franks, Ana Sendova-Franks, Tom Richardson, and Elva Robinson provided comments and discussion on the manuscript. This work was supported by a National Science Foundation Minority Postdoctoral Fellowship.

Open Access This article is distributed under the terms of the Creative Commons Attribution Noncommercial License which permits any noncommercial use, distribution, and reproduction in any medium, provided the original author(s) and source are credited.

\section{References}

Adami C (2002) What is complexity? Bioessays 24:1085-1094

Aleksic Z (2000) Artificial life: growing complex systems. In: Bossomaier RJ, Green DG (eds) Complex systems. Cambridge University Press, Cambridge, pp 91-126 
Anderson PW (1972) More is different-broken symmetry and the nature of the hierarchical structure of science. Science 177:393

Balleine BW, Killcross S (2006) Parallel incentive processing: an integrated view of amygdala function. Trends Neurosci 29:272-279

Bar-Yam Y (2004) A mathematical theory of strong emergence using multiscale variety. Complexity 9:15-24

Berent I, Pinker S, Tzelgov J, Bibi U, Goldfarb L (2005) Computation of semantic number from morphological information. J Mem Lang 53:342-356

Bonabeau E, Theraulaz G, Deneubourg JL, Aron S, Camazine S (1997) Self-organization in social insects. Trends Ecol Evol 12:188-193

Camazine S, Deneubourg J, Franks NR, Sneyd J, Theraulaz G, Bonabeau E (2001) Self-organization in biological systems. Princeton University Press, Princeton

Corning PA (2002) The re-emergence of "emergence": a venerable concept in search of a theory. Complexity 7:18-30

Coveney PV (2003) Self-organization and complexity: a new age for theory, computation and experiment. Philos Trans R Soc A 361:1057-1079

Crutchfield JP (1994) The calculi of emergence: computation, dynamics and induction. Phys D 75:11-54

Crutchfield JP, Young K (1989) Inferring statistical complexity. Phys Rev Lett 63:105-108

Dehaene S, Spelke E, Pinel P, Stanescu R, Tsivkin S (1999) Sources of mathematical thinking: behavioral and brain-imaging evidence. Science 284:970-974

Denuebourg JL, Goss S (1989) Collective patterns and decision making. Ethol Ecol Evol 12:188-193

Dieck ST, Brandstatter JH (2006) Ribbon synapses of the retina. Cell Tissue Res 326:339-346

Gardner M (1970) Fantastic combinations of John Conway's new solitaire game life. Sci Am 223:120-123

Gardner M (1971) Cellular automata, self-reproduction, Garden of Eden and Game life. Sci Am 224: $112-117$

Halley JD, Winkler DA (2006) Classification of self-organization and emergence in chemical and biological systems. Aust J Chem 59:849-853

Nehaniv CL, Rhodes JL (2000) The evolution and understanding of hierarchical complexity in biology from an algebraic perspective. Artif Life 6:45-67

Newth D, Finnigan J (2006) Emergence and self-organization in chemistry and biology. Aust J Chem 59:841-848

Nicolis G, Prigogine I (1989) Exploring complexity. W.H. Freeman, New York

Pinker S (1997) How the mind works. Norton \& Company, New York

Prigogine I (1980) From being to becoming: time and complexity in the physical sciences. W. H. Freeman, New York

Ramachandran VS, Blakeslee S (1998) Phantoms in the brain: probing the mysteries of the human mind. William Morrow, New York

Russell B (1945) A history of western philosophy. Simon and Schuster, New York

Spehr M, Spehr J, Ukhanov K, Kelliher KR, Leinders-Zufall T, Zufall F (2006) Parallel processing of social signals by the mammalian main and accessory olfactory systems. Cell Mol Life Sci 63:1476-1484

Turing AM (1952) The chemical basis of morphogenesis. Philos Trans R Soc B 237:37-72

Vicsek T (2002) Complexity-the bigger picture. Nature 418:131-131

von Frisch K (1967) The dance language and orientation of bees. Harvard University Press, Cambridge

Wolfram S (1984a) Cellular automata as models of complexity. Nature 311:419-424

Wolfram S (1984b) Universality and complexity in cellular automata. Phys D 10:1-35

Wolfram S (2002) A new kind of science. Wolfram Media, Champaign 